What the new map shows is that the thickness of the lithosphere varies from a few tens of kilometres in young oceans and continental orogenic provinces to more than $300 \mathrm{~km}$ in shield areas. It is also evident from the analysis which led to the production of the map that beneath the continental shields the asthenosphere is very thin. Indeed, a strict interpretation would suggest that the asthenosphere does not exist there at all, for shield geotherms do not intersect the solidus at any depth, although shield lithosphere is presumably partially decoupled from the deeper interior at the depth at which the appropriate geotherm approaches the solidus most closely. In any event, there is a clear implication that the viscous drag at the base of shield lithosphere is much higher than elsewhere-a prediction which receives some support from observation.

Finally, it is clear that the thickness of the lithosphere, both continental and oceanic, increases (not linearly) with age. This implies that shield lithosphere was once much thinner than it is now and that the asthenosphere beneath it was thicker, thus facilitating continental drift during the Precambrian. As for the future, continental lithosphere will presumably continue to thicken, increasing the viscous drag at its base and thus graually bringing drift to a halt.

\section{Communal nesting in birds}

\section{from John Krebs}

ThE Central and South American Groove-billed Ani (Crotophaga sulcirostus) lives in groups of one to four monogamous pairs. All the females in a group lay their eggs in a communal nest, and both members of each pair help to incubate and feed the young. The axiom of selfish genery warns one to expect that Ani groups are uneasy alliances in which each individual pursues its own reproductive ends. A study recently reported by $\mathbf{S}$. L. Vehrencamp (Science 197, 403; 1977) reveals that this is indeed the case. The most obvious and unequivocal sign of self interest is that the females in a group roll each other's eggs out of the communal nest. Females cannot, however, distinguish their own eggs from those of others so that an individual only engages in egg-rolling before she herself has started to lay. This means, of course, that the first female to start

John Krebs is at the Edward Grey Institute of Field Ornithology at the University of Oxford. laying eggs is more likely to suffer, and the last female to start loses no eggs. Although the first female(s) to lay to some extent compensate for egg loss by laying larger chutches, they still end up with fewer eggs than the last female at the time of incubation. In comparing nesting groups of different sizes, Vehrencamp found that the average number of eggs per female is positively correlated with group size, but the first female to lay in the group raises fewer young than a solitary pair, while the last female raises more. So far all the benefits seem to lie with the last female to lay and none with the first. There is potentially a cost to the late layer, because the first female starts to incubate as soon as she has completed her clutch, and the first young to hatch out survive better than later ones. The late female's answer to this is to lay her eggs more rapidly, at 1-2 day intervals compared with 2-3 days for early females, so that her young hatch out no later than average. All in all, the late females clearly do better than early layers, and they tend to be older (probably more dominant) individuals who are mated to larger than average males.

The late layers also gain when it comes to parental care: they contribute less to incubation and feeding the young than do the other females in a group even though most of the brood is theirs. This picture of more or less parasitic behaviour by late females is to some extent ameliorated by the fact that the males mated to late layers do more incubation and feeding of the young than any other group member. This implies that the male can tell that his mate has laid most of the successful eggs so that he is feeding his own children.

Clearly we need to know a lot more about the Ani system of communal nesting before a full cost benefit analysis for each group member can be done. At the moment it looks as though the late-laying female and her mate get more out of the system than the others, so further data are needed to work out why the communal nesting system is a stable, if selfish alliance.

In other communal nesting species which have been studied over several years, the members of a group are usually closely related to one another. However, these species, including babblers of the genus Turdoides and two American jays (Aphelocoma spp.), have a different system of communal nesting from that of the anis. Only one pair does all the reproducing, and the other group members are usually subadults, many of them offspring of the reproductive pair and hence siblings of the present nestlings. Although the helpers, as they are called, may in- dators, and feed nestlings, there is some uncertainty about how much they actually increase the reproductive success of the breeding pair (Zahavi Ibis 116, 84; 1974; Woolfenden Auk 92, 1; 1975; Gaston, thesis, Oxford, Univ. 1976). One interpretation (Gaston op. cit.) is that the helpers are providing just enough aid to encourage the parents to allow them to stay in their territory. In both the babblers and jays, young birds have rather little chance of establishing their own territory away from home, because the suitable habitat is fully occupied by adults, who have a low mortality rate. The best bet for a young bird is to stay at home and try to take over the parental territory when one or both of the parents die.

In doing this, the young birds could reduce the future reproductive success of their parents by competing for food, attracting predators to the nest, and so on. In order to offset this, and hence make it profitable for the parents to allow them to stay, they provide a small amount of help in rearing new young.

This interpretation is speculative, but it emphasises that the apparently simple cooperation probably has a rich and fascinating underlying pattern of selfish behaviour

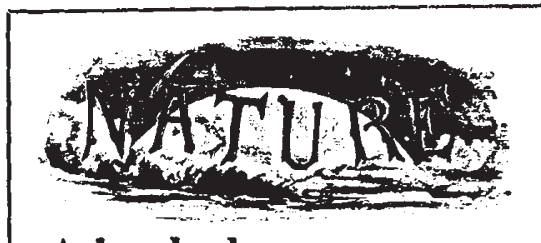

\section{A hundred years ago}

METEOROLOGY AND THE INDIAN Famine... Dr Hunter also found that six great scarcities of sufficient gravity to be officially returned as "famines" had occurred during the period 1880-77. Of these six famines five were caused by years of drought coincident with, or adjoining to, the periods of minimum sunspots. He further showed that the rainfall at Madras passed through an eleven year's cycle, corresponding with the cycle of sun-spots. That is to say, the rainfall reaches its minimum in the eleventh year, rises to its maximum about half-way through the cycle in the fifth year, and then declines again to its minimum in the eleventh year.

At the recent biennial meeting of the German Astronomical Society, which was held at Stockholm, the members received the news of the discovery of the two satellites of Mars with manifestations of grave doubts. The president at their request telegraphed to the Berlin Observatory, and in reply received a copy of the original telegram as it was sent from America.

From Nature 16, 13 September, 425, $428 ; 1877$. 